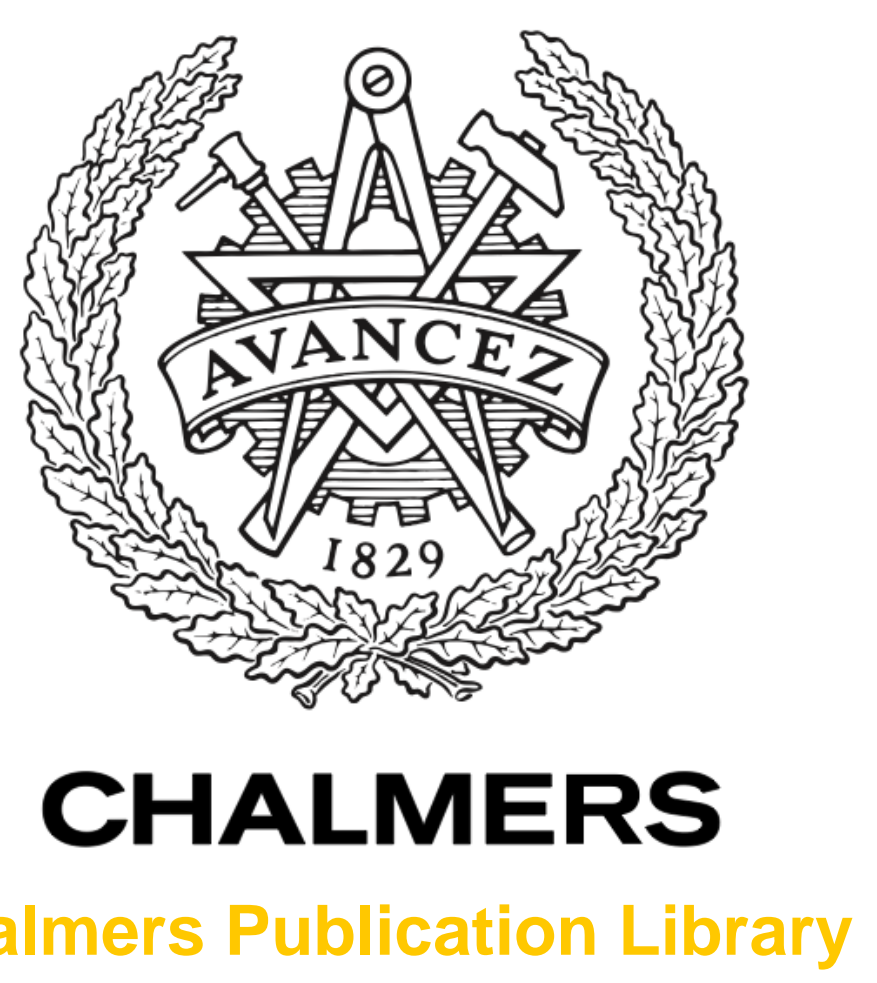

Chalmers Publication Library

\title{
Performance Comparison of Differential 8-Ary Modulation Formats in High-Speed Optical Transmission Systems
}

This document has been downloaded from Chalmers Publication Library (CPL). It is the author's version of a work that was accepted for publication in:

Journal of Lightwave Technology (ISSN: 0733-8724)

Citation for the published paper:

Tipsuwannakul, E. ; Johannisson, P. ; Sköld, M. (2011) "Performance Comparison of Differential 8-Ary Modulation Formats in High-Speed Optical Transmission Systems". Journal of Lightwave Technology, vol. 29(19), pp. 2954-2962.

http://dx.doi.org/10.1109/JLT.2011.2163495

Downloaded from: http://publications.lib.chalmers.se/publication/146728

Notice: Changes introduced as a result of publishing processes such as copy-editing and formatting may not be reflected in this document. For a definitive version of this work, please refer to the published source. Please note that access to the published version might require a subscription.

Chalmers Publication Library (CPL) offers the possibility of retrieving research publications produced at Chalmers University of Technology. It covers all types of publications: articles, dissertations, licentiate theses, masters theses, conference papers, reports etc. Since 2006 it is the official tool for Chalmers official publication statistics. To ensure that Chalmers research results are disseminated as widely as possible, an Open Access Policy has been adopted.

The CPL service is administrated and maintained by Chalmers Library. 


\title{
Performance Comparison of Differential 8-Ary Modulation Formats in High-Speed Optical Transmission Systems
}

\author{
Ekawit Tipsuwannakul, Pontus Johannisson, Mats Sköld, Erik Agrell, Magnus Karlsson, and \\ Peter A. Andrekson, Fellow, IEEE
}

\begin{abstract}
This paper presents back-to-back and transmission performance comparisons between single-polarization $120 \mathrm{Gbit} / \mathrm{s}$ RZ-DQP-ASK and RZ-D8PSK formats in a single-wavelength system. In the back-to-back configuration, we determine the OSNR requirements of the two formats and compare the results with those obtained analytically and numerically. In the transmission experiments, we examine signal constellations to quantify the significance of intra-channel fiber nonlinearities and investigate the BER performance of the two formats over different reaches (up to $480 \mathrm{~km}$ ). The results show that the performance of DQP-ASK is superior to D8PSK in back-to-back configuration but is inferior over long distances.
\end{abstract}

Index Terms-ASK, D8PSK, differential detection, DQP-ASK, DQPSK, fiber nonlinearities, IFWM, modulation format, optical transmission, SPM.

\section{INTRODUCTION}

D IFFERENTIAL detection is regarded as a scheme for relatively short-range high-speed transmission systems, e.g., metro-networks as it provides low receiver complexity and cost. The scheme is capable of detecting not only differential phase shift keying (DPSK) but also differential-phase and amplitude shift keying (DP-ASK) modulation formats and, at the same time, offers a feasible solution for near-term realization [1]-[9].

As a result, both DPSK and DP-ASK formats carrying (up to) $4 \mathrm{bit} / \mathrm{symbol}$ have been demonstrated at high symbol rates over the past several years. For instance, Gnauck et al. [1] reported a 1-bit/symbol format, binary DPSK (DBPSK), with the symbol rate of $43 \mathrm{Gbaud}$ (corresponding to $86 \mathrm{Gbit} / \mathrm{s}$ in the polarization multiplexed system). Furthermore, B. Koch et al. [2], [3] presented 50 Gbaud quadrature DPSK (DQPSK) carrying 2 bit/symbol per polarization with the aggregate bit-rate of 200

Manuscript received May 11, 2011; revised June 23, 2011; accepted July 26, 2011.

This work was supported by the Swedish Foundation for Strategic Research (SSF) and Swedish Governmental Agency for Innovation Systems (VINNOVA) within the 100 GET program.

E. Tipsuwannakul, P. Johannisson, M. Karlsson, and P. A. Andrekson are with the Photonics Laboratory, Department of Microtechnology and Nanoscience (MC2), Chalmers University of Technology, SE-412 96 Gothenburg, Sweden (e-mail: ekawit@chalmers.se).

M. Sköld is with EXFO Sweden AB, SE-411 33 Gothenburg, Sweden.

E. Agrell is with the Department of Signals and Systems (S2), Chalmers University of Technology, SE-412 96 Gothenburg, Sweden.

Color versions of one or more of the figures in this paper are available online at http://ieeexplore.ieee.org.

Digital Object Identifier 10.1109/JLT.2011.2163495
Gbit/s. Moreover, 3-bit/symbol formats, 8-ary DP-ASK (DQPASK) and 8-ary DPSK (D8PSK), were shown in [4]-[7], both achieving the same record bit-rate of $240 \mathrm{Gbit} / \mathrm{s}$ over a single wavelength with the corresponding symbol rate of 40 Gbaud. A higher-order format such as 16-level DP-ASK (4 bit/symbol) has also been reported but, due to its complexity, the symbol rate was limited to 10 Gbaud [8].

DQP-ASK and D8PSK are, in fact, similar in many ways. For instance, both formats carry $3 \mathrm{bit} / \mathrm{symbol}$, occupy similar spectral width, and have comparable transmitter/receiver complexity. However, since the symbols of the two formats are distributed differently, it is difficult to speculate which format would provide the better performance after transmission limited by fiber nonlinear effects. On one hand, D8PSK is likely to suffer more from fiber nonlinearities since the angular distance between symbols (in the constellation) is half of that of DQP-ASK and thus more susceptible to nonlinear distortion. On the other hand, DQP-ASK is expected to experience severe nonlinear degradation as the format contains two different intensity levels. Evaluating the performance of the two modulation formats over various transmission distances is, thus, essential to determine the most appropriate one for certain applications.

In this paper, we comprehensively compare the back-to-back and transmission performances of single-wavelength, single-polarization 40 Gbaud return-to-zero (RZ)-DQP-ASK and RZ-D8PSK over various distances (up to $480 \mathrm{~km}$ experimentally). To our knowledge, this, together with [10], is the first direct comparison of two differential 8-ary formats and also the first study of the influence of fiber nonlinearities on DQP-ASK at 40 Gbaud. In the back-to-back configuration, we analytically, numerically, and experimentally quantify the required optical signal-to-noise ratio (OSNR) of the two formats and, in the case of DQP-ASK, further determine the ratio between the two intensity levels that gives the optimum system performance. For transmission limited by intra-channel nonlinear effects, we investigate the distortion of the two modulation formats using signal constellations obtained numerically and experimentally. We also quantify the optimal launch power $\left(\mathrm{P}_{\mathrm{L}}\right)$, which is a trade-off between noise and fiber nonlinearities, of the formats over various distances and plot the bit-error-rate (BER) (at the optimal launch power) as a function of the transmission reach. The results reveal that either format can be superior over different distances. It should be noted that the two signals evaluated here have an aggregated bit-rate of $120 \mathrm{Gbit} / \mathrm{s}$, 


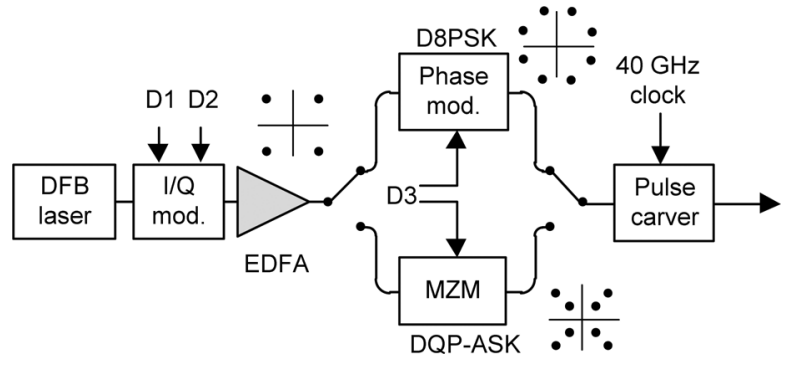

Fig. 1. 40 Gbaud RZ-DQP-ASK/D8PSK transmitter implemented in the experimental setup.

which is highly relevant for forthcoming 100 Gigabit Ethernet implementations.

This paper is organized as follows. In Section II, we describe the detailed setup of the transmitter, the receiver, and the transmission link used in the experiments. In Section III, we expound the numerical system implementation, which is generally modeled to replicate that implemented in the experiments. In Section IV, we derive the back-to-back performance of the two formats as a function of OSNR and compare with those obtained from the simulated and the actual systems. Finally, in Section V, we numerically and experimentally evaluate the influence of fiber nonlinearities on the two formats by means of signal constellations and the BER.

\section{EXPERIMENTAL SETUP}

As illustrated in Fig. 1, the RZ-DQP-ASK/RZ-D8PSK transmitter consisted of a $1 \mathrm{MHz}$ linewidth distributed-feedback (DFB) laser, a $31 \mathrm{GHz} \mathrm{I} / \mathrm{Q}$ modulator, a $35 \mathrm{GHz}$ phase modulator (PM), and two chirp-free Mach-Zehnder modulators (MZMs). The I/Q modulator was driven by two of $40 \mathrm{Gbit} / \mathrm{s}$ binary data streams (D1 and D2), providing $80 \mathrm{Gbit} / \mathrm{s}$ DQPSK data at the output. The DQPSK signal was amplified with an Erbium-doped fiber amplifier (EDFA) and the amplified signal was fed into either an MZM or a PM that was driven by the third $40 \mathrm{Gbit} / \mathrm{s}$ binary data stream (D3). The former modulator added amplitude modulation while the latter created a $\pi / 4$ phase shift onto DQPSK for DQP-ASK and D8PSK generation, respectively. Finally, an MZM was then driven by a sinusoid to generate a RZ format with $50 \%$ duty cycle. All data streams (D1, D2, and D3) used in this work were decorrelated pseudo random bit sequences (PRBSs) with the length of $2^{11}-1$. The length of the PRBS was essentially limited by the programming capability of an error detector (ED). To obtain a fair comparison, the OSNR and the optical power of the two signals at the transmitter output were adjusted to be approximately $52 \mathrm{~dB}$ and $-15 \mathrm{dBm}$, respectively. This was done by varying the optical power before and after the EDFA via variable attenuators. The measured constellations of the two modulation formats generated from the transmitter are depicted in Fig. 2 while the measured signal spectra are illustrated in Fig. 3. The larger spreading of symbol points seen in the experimental constellations (compared with those from the simulation) is due to the imperfections of the driving signals such as noise and data jitter (originating from signal generation) that were not accounted for in the simulations. Notice that the

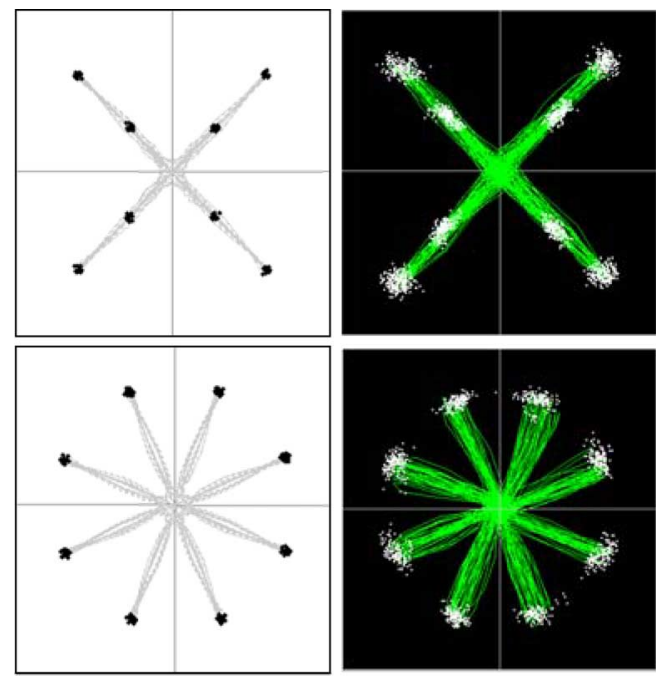

Fig. 2. (left) Simulated and (right) measured signal constellationa of $40 \mathrm{Gbaud}$ (top) RZ-DQP-ASK and (bottom) RZ-D8PSK signals from the transmitter. The measured contellations had the OSNR of the $52 \mathrm{~dB}$ and were capture with EXFO modulation analyzer PSO200.
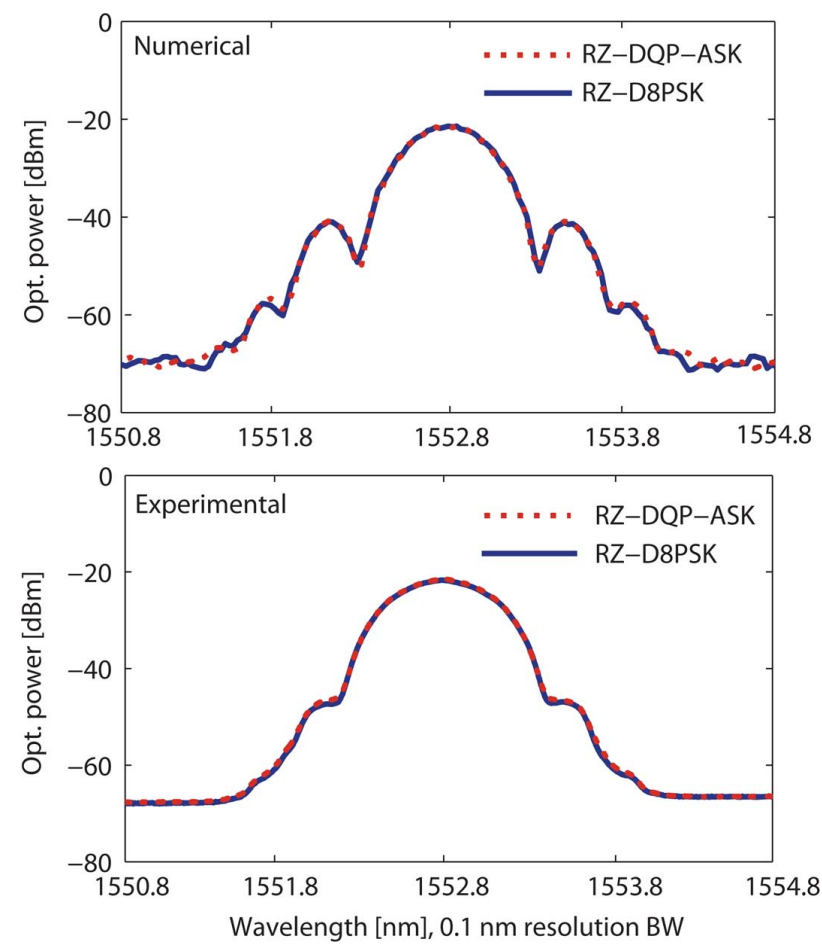

Fig. 3. Simulated (top) and measured (bottom) signal spectra of 40 Gbaud RZ-DQP-ASK (dotted lines) and RZ-D8PSK (solid lines) signal from the transmitter.

experimental signal spectra plotted in Fig. 3 are broader than those obtained numerically. We believe it is because the duty cycle of the RZ format used in the experiment is slightly lower than $50 \%$.

Fig. 4 illustrates the $480 \mathrm{~km}$ transmission link used in the experiment. The link was realized by six spans, each containing an $80 \mathrm{~km}$ standard single mode fiber (SMF) and double-stage EDFAs with a $12 \mathrm{~km}$ dispersion compensating fiber (DCF) in between. The average loss of the SMFs and DCFs in each span were measured to be 18 and $12 \mathrm{~dB}$, respectively. The chromatic 


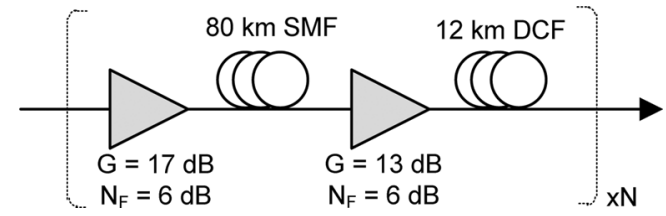

Fig. 4. Transmission link block diagram.

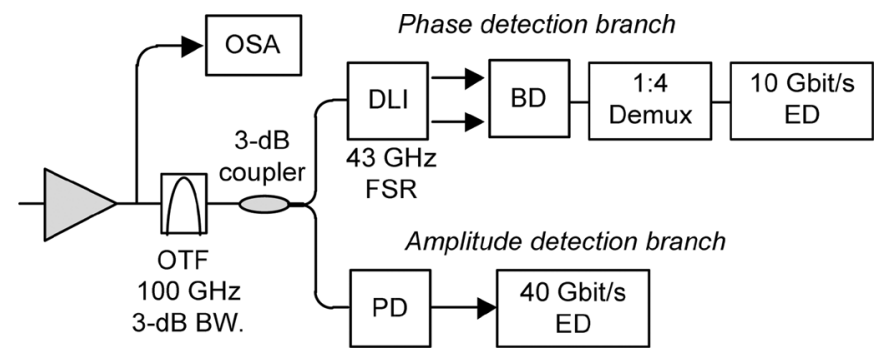

Fig. 5. 40 Gbaund DQP-ASK/D8PSK receiver implemented in the experimental setup. OSA denotes an optical spectrum analyzer.

dispersion at the operating wavelength of the SMFs and the DCFs is found to be approximately 17 and $-114 \mathrm{ps} /(\mathrm{nm} \mathrm{km})$, yielding small residual dispersion after each span. Short pieces of the SMF were then used after the link to achieve zero net dispersion. The launch power into the DCFs was also kept $5 \mathrm{~dB}$ lower than that into SMFs to reduce the impact of fiber nonlinearities in the DCF [11].

At the receiver (as shown in Fig. 5), an optical tunable filter (OTF) with the $3-\mathrm{dB}$ bandwidth of $100 \mathrm{GHz}$ was used to reduce the amplified spontaneous emission (ASE) noise and the filtered signal was split into two branches by a 3-dB coupler for amplitude and phase detection. For the phase detection, the signal was differentially demodulated by a delay line interferometer (DLI) with a free spectral range (FSR) of $43 \mathrm{GHz}$ (non-ideal) and the demodulated signal was detected by a $50 \mathrm{GHz}$ balanced detector (BD). The detected signals were then electronically demultiplexed by a 1:4 demultiplexer and fed into an ED, which was programmed with the expected differentially demodulated bit patterns due to the lack of a differentially pre-coder. Note that the use of the DLI with the non-ideal FSR gives rise to distortion of the differentially demodulated signal. However, such degradation has been shown to be negligible when the FSR differs less than $10 \%$ from the ideal value as is in this work [12].

For the amplitude detection, the signal was directly detected by a $50 \mathrm{GHz}$ photodetector (PD) and the detected signal was fed into a $40 \mathrm{Gbit} / \mathrm{s} \mathrm{ED}$. The detected signal eye diagrams from the two branches in the back-to-back configuration are illustrated in Fig. 6. Note that the approximate 2 ps skew seen in the eye diagrams of the phase tributaries is caused by a signal path length mismatch of the $\mathrm{BD}$.

\section{NUMERICAL MODEL}

In the transmitter, the DFB laser was modeled as an ideal light source while the influence of the modulator bandwidth was emulated by low-pass filtering the driving signals with the 5th-order Bessel filter with the bandwidth comparable to those of the modulators used in the experiment. Similarly to the experiment, the driving signals were decorrelated PRBSs with

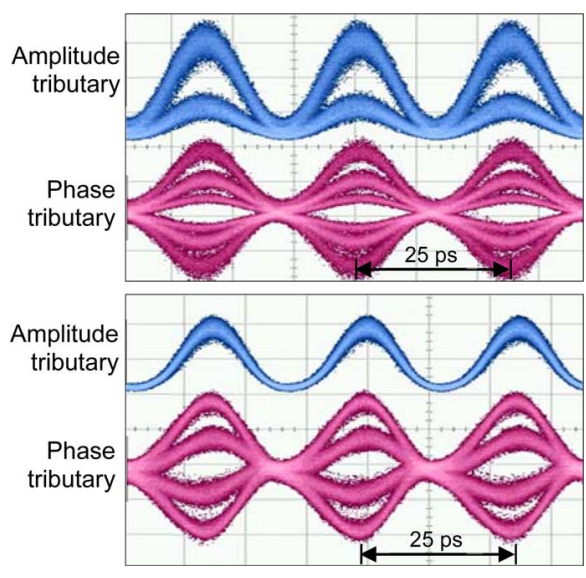

Fig. 6. Experimentally delected signals from amplitude and phase tributaries of (top) 40 Gbaud RZ-DQP-ASK and (bottom) RZ-D8PSK signals.

TABLE I

Lists OF Fiber PARAMETERS IMPLEMENTEd IN THE SiMULATED SySTEM

\begin{tabular}{lll}
\hline \hline & SMF & DCF \\
\hline$\gamma\left[\mathrm{W}^{-1} \mathrm{~km}^{-1}\right]$ & 1.41 & 2.83 \\
$D[\mathrm{ps} /(\mathrm{nm} \mathrm{km})]$ & 16.5 & -110 \\
$\alpha[\mathrm{dB} / \mathrm{km}]$ & 0.225 & 1.00 \\
Length $[\mathrm{km}]$ & 80.0 & 12.0 \\
\hline \hline
\end{tabular}

the length of $2^{11}-1$. At the output of the transmitter, the OSNR and the power of the optical signal were adjusted to be $52 \mathrm{~dB}$ and $-15 \mathrm{dBm}$, respectively. The constellations and the optical spectra of 40 Gbaud RZ-DQP-ASK and RZ-D8PSK signals simulated from this transmitter are depicted in Figs. 2 and 3.

To model light propagation in an optical fiber, we use the nonlinear Schrödinger equation

$$
\frac{\partial A}{\partial z}+\frac{\alpha}{2} A+\frac{i \beta_{2}}{2} \frac{\partial^{2} A}{\partial T^{2}}=i \gamma|A|^{2} A
$$

where $A$ denotes the complex amplitude of the field envelope, $\alpha$ is the attenuation factor of the fiber, $\gamma$ corresponds to the Kerr nonlinearity coefficient, and $\beta_{2}$ designates the group-velocity dispersion parameter. As explained in [13], this model neglects the influence of higher-order dispersion, fiber birefringence, intra-pulse Raman scattering, and self-steepening. The equation was solved using the split-step Fourier method.

In this simulation, we modeled the inline EDFAs as noise-free amplifiers and the ASE noise was later added directly before the receiver, which reduces the computational time significantly. By doing so, the nonlinear signal-noise interaction is ignored. However, this nonlinear process has been shown to be minor in the systems operating at high symbol rates and over short distances as is the case in this work [14]. The amount of noise added before the receiver was adjusted to be the same as is in the experiments. The fiber parameters used in the simulation are shown in Table I.

At the receiver, the optical filter was a 4th-order super-Gaussian function with the 3-dB bandwidth of $100 \mathrm{GHz}$. The FSR of the DLI is set to $43 \mathrm{GHz}$, similar to that used in the experiment. In addition, The BER of all signal tributaries was obtained from Monte Carlo simulations by direct error 
a)

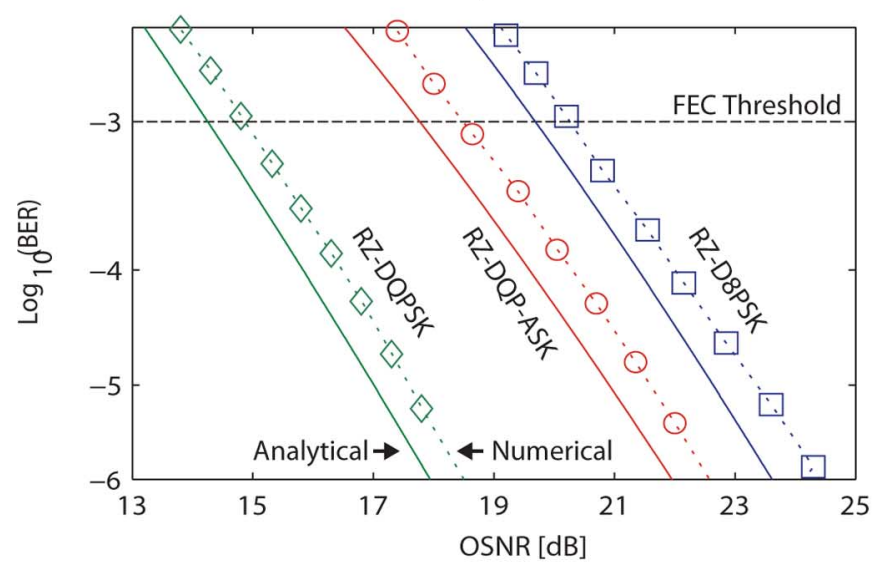

b)

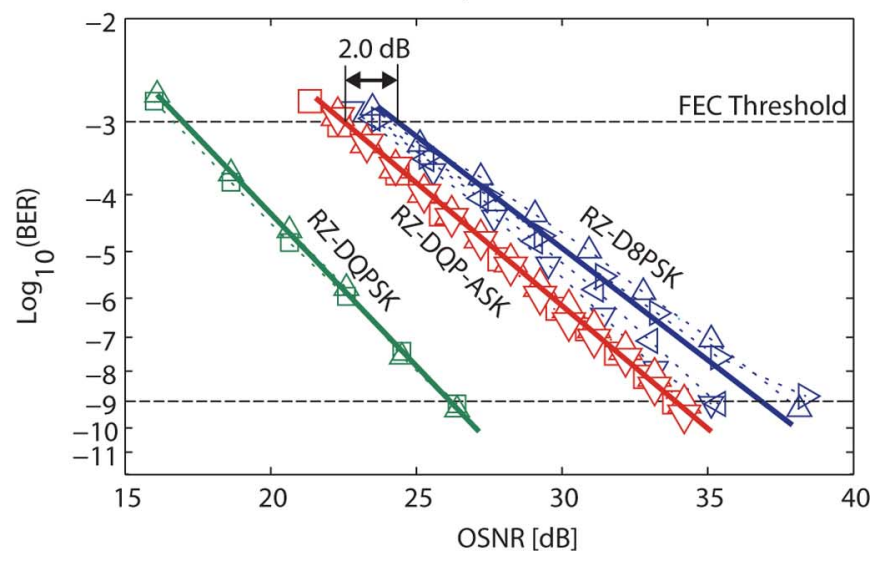

Fig. 7. (top) Analytical, numerical and (bottom) experimental back-to-back performance of 40 Gbaud RZ-DQPSK/D8PSK signals as a function of the OSNR.

counting. It should be noted that the simulated system is assumed to be ASE noise-limited and we ignored the influence of thermal and shot noise. For the OSNR measurement, we take into account the ASE noise power from both orthogonal polarizations.

\section{BACK-TO-BACK PERformanCE}

In this section, we first derive the theoretical limit of 40 Gbaud RZ-DQP-ASK and RZ-D8PSK, and then compare with the simulated and measured results. The analytical and numerical results of the two formats are plotted in Fig. 7(a) while those from the experiment are shown in Fig. 7(b). The BER performance of RZ-DQPSK at the same symbol rate is also included in the plots as a benchmark.

\section{A. Analytical BER Performance}

The SER for M-PSK with differential detection in the presence of additive white Gaussian noise is [15]

$$
\operatorname{SER}(\gamma, M)=\frac{\sin (\pi / M)}{2 \pi} \int_{-\pi / 2}^{\pi / 2} \frac{e^{-\gamma[1-\cos (\pi / M) \cos t]}}{1-\cos (\pi / M) \cos t} d t
$$

where

$$
\gamma=\left\langle E_{s}\right\rangle / N_{0} .
$$

In these equations, $\left\langle E_{s}\right\rangle$ is the average energy per symbol and $N_{0}$ is the noise power spectral density (PSD). By assuming the use of Gray coding, we have

$$
\mathrm{BER} \approx \mathrm{SER} / \log _{2} M .
$$

Assuming single polarization transmission, we can relate $\gamma$ to the OSNR using the expression

$$
\operatorname{OSNR}=\frac{\left\langle E_{s}\right\rangle R_{s}}{2 N_{0} \Delta \nu_{0.1} \mathrm{~nm}}=\frac{R_{s}}{2 \Delta \nu_{0.1 \mathrm{~nm}}} \gamma
$$

where $R_{s}$ is the symbol rate and $\Delta \nu_{0.1} \mathrm{~nm}$ is the reference bandwidth $(0.1 \mathrm{~nm})$. With the bit-to-symbol mapping used here, a DQP-ASK symbol can be considered to carry two "phase bits" and one "amplitude bit". In order to find the BER for DQP-ASK, we treat these two cases, i.e., the DQPSK receiver and the direct-detection receiver, separately and then combine the two results to obtain the average BER.

As seen in Fig. 6, the differentially demodulated eye diagram is split up into $2 \times 3$ amplitude levels when detecting the phase bits of DQP-ASK. The dominating part of the errors is caused by the innermost levels and, in order to use (2), we need to use the reduced symbol energy that corresponds to this level. Denoting the two amplitude levels of DQP-ASK by $a$ and $b$ (with $a<b$ ), we obtain

$$
\gamma_{\mathrm{red}}=\frac{\left\langle E_{s}\right\rangle}{N_{0}} \frac{a^{2}}{\left(a^{2}+b^{2}\right) / 2}=\frac{2 \eta}{1+\eta} \gamma
$$

where the intensity ratio is defined $\eta=a^{2} / b^{2}$. From this equation, we notice that $\gamma_{\mathrm{red}} \rightarrow \gamma$ as $\eta \rightarrow 1$. The detected current is on the innermost levels when two consecutive low-power symbols are transmitted, and on average this occurs in one case out of four. The SER for the phase bits is therefore

$$
\operatorname{SER}_{\text {phase }}=\operatorname{SER}\left(\gamma_{\mathrm{red}}, M=4\right) / 4 .
$$

Assuming Gray coding, we obtain

$$
\mathrm{BER}_{\text {phase }} \approx \mathrm{SER}_{\text {phase }} / 2 .
$$

For optimal performance in the direct detection, we assume that a matched optical filter is used. The incoming optical field consists of a signal part and complex white Gaussian noise and since the detected current is proportional to the intensity, the current will have a noncentral chi-square distribution [16]. The optimal decision threshold for the current is the intersection of the probability density functions corresponding to the high and low amplitude levels, respectively, and can be found numerically. The cumulative distribution function is expressed using the Marcum $Q$ function, see [16]. Using (6) and the fact that the noise variance is $N_{0} / 2$ per real dimension, we obtain

$$
\begin{aligned}
\mathrm{BER}_{\mathrm{amp}}=\left[1+Q\left(\sqrt{2 \gamma_{\mathrm{red}}},\right.\right. & \sqrt{\xi \gamma}) \\
& \left.-Q\left(\sqrt{2 \gamma_{\mathrm{red}} / \eta}, \sqrt{\xi \gamma}\right)\right] / 2
\end{aligned}
$$


where the parameter $\xi$ is chosen numerically to minimize $\mathrm{BER}_{\mathrm{amp}}$. The minimal BER $\mathrm{Bmp}_{\mathrm{amp}}$ is affected by $\eta$, with optimum performance as $\eta \rightarrow 0$. This shows that the choice of the intensity ratio is a trade-off between the phase and amplitude bits. The average BER can be set up using $\mathrm{BER}_{\text {phase }}$ and $\mathrm{BER}_{\mathrm{amp}}$ according to

$$
\mathrm{BER}=\frac{2}{3} \mathrm{BER}_{\mathrm{phase}}+\frac{1}{3} \mathrm{BER}_{\mathrm{amp}} .
$$

In Fig. 7, we have plotted the BER as a function of the OSNR at 40 Gbaud for DQPSK, D8PSK, and DQP-ASK. The intensity ratio that minimizes the OSNR requirement at $\mathrm{BER}=$ $10^{-3}$ has been used. It is found that the minimum OSNR is $17.8 \mathrm{~dB}$ and occurs for $\eta=0.205$ (corresponding to $6.88 \mathrm{~dB}$ ), For DQPSK and D8PSK, the required OSNR is $14.3 \mathrm{~dB}$ and $19.7 \mathrm{~dB}$, respectively.

The optimum intensity ratio depends on the SNR and $\eta$ will increase with the SNR. The asymptotic value for $\eta$ at high SNR can be found by approximating the exact expression, but can also be found from single scaling arguments. Thus, in the limit of high SNR, we have [15]

$$
\mathrm{BER}_{\text {phase }} \propto \operatorname{erfc} \sqrt{a^{2}[1-\cos (\pi / 4)] / N_{0}} .
$$

In the same limit [15]

$$
\mathrm{BER}_{\mathrm{amp}} \propto \operatorname{erfc}\left[(b-a) /\left(2 \sqrt{N_{0}}\right)\right]
$$

By requiring the arguments of the erfc function to be equal, we directly obtain $\eta=0.231(6.37 \mathrm{~dB})$.

\section{B. Discussion of the Results}

We first computed/measured the error rates corresponding to binary decision thresholds of all amplitude and/or phase tributaries individually. Each phase tributary was measured separately by applying phase offsets to one arm of the DLI $( \pm \pi / 4$ for DQP-ASK, and $\pm \pi / 8, \pm 3 \pi / 8$ for D8PSK), yielding 3 (including the amplitude tributary) and 4 different error rates. By assuming that Gray coding is implemented and that symbol errors only occur between neighboring symbols, the BER of the system is then, assuming the average of these error rates in the case of DQP-ASK [3], [4] and the average multiplied by 4/3 in the case of D8PSK [17], [18].

As can be seen from Fig. 7(a), the numerical results of M-ary DPSK signals are consistent with the previous work [19] but approximately $0.6 \mathrm{~dB}$ worse than the analytical expectation (including DQP-ASK), which we attribute to non-matched filtering in the simulated receiver. In the case of DQP-ASK, the numerical optimum ratio of the two intensity levels obtained from the simulated system is found to be $6.8 \mathrm{~dB}$, which is also in good agreement with the analytical solution. In addition, the numerical results show approximately $1.8 \mathrm{~dB}$ superior performance in the case of DQP-ASK to that of D8PSK for BER $=10^{-3}$.

The experimental results shown in Fig. 7(b) also illustrate a similar difference $(\sim 2 \mathrm{~dB})$ between the performance of DQP-ASK and D8PSK despite the fact that the measured required OSNR is higher than those from the simulated system. However, in the case of experimental DQP-ASK, the optimum ratio of the two intensity levels is found to be $4.7 \mathrm{~dB}$. Although
TABLE II

THEORETICAL, NUMERICAL, AND EXPERIMENTAL REQUIRED OSNR (IN DB) FOR BER $=10^{-3}$ OF THREE DIFFERENT FORMATS AT 40 GBAUD

\begin{tabular}{rccc}
\hline \hline & Theoretical & Numerical & Experimental \\
\hline RZ-DQPSK & 14.3 & 14.9 & 17.0 \\
RZ-DQP-ASK & 17.8 & 18.5 & 22.5 \\
RZ-D8PSK & 19.7 & 20.3 & 24.5 \\
\hline \hline
\end{tabular}

a)

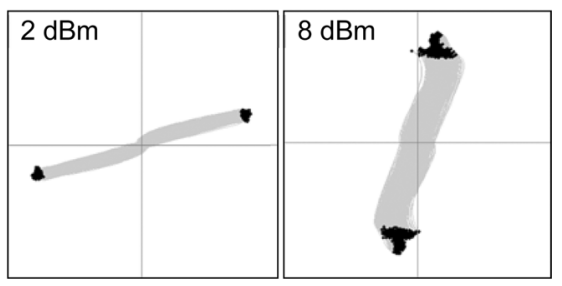

b)

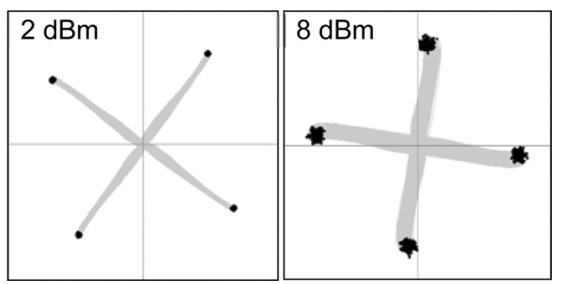

Fig. 8. Simulated noise-free constellations of (a) 40 Gbaud RZ-DBPSK and (b) RZ-DQPSK signals over $320 \mathrm{~km}$ with (left) $2 \mathrm{dBm}$ and (right) $8 \mathrm{dBm}$ launch power.

approximately $2 \mathrm{~dB}$ deviated from the theory, it is consistent with that experimentally reported in [3], [4]. The difference between the analytical and experimental optimum ratio indicates that the experimental phase tributaries of DQP-ASK perform slightly worse than ideal. The reason is imperfections in the delay interferometer, the $\mathrm{BD}$, and the electrical demultiplexer, causing an extra penalty on the phase tributaries.

Table II summarizes the analytical, numerical, and experimental OSNRs required for BER $=10^{-3}$ of the three modulation formats. In the case of DQPSK, the OSNR obtained experimentally is roughly $3 \mathrm{~dB}$ higher than that found analytically, which is mainly due to the amplitude distortion and data jitter associated with the electrical driving signal generation. This penalty increases to approximately $5 \mathrm{~dB}$ in the case of DQP-ASK and D8PSK, which we attribute, at least to a large extent, to the use of an additional driving signal in the transmitter. It should be noted that the predetermined $\mathrm{BER}=10^{-3}$ can achieve the corrected $\mathrm{BER}=10^{-15}$, provided that the forward error correction overhead with enhanced Reed-Solomon with concatenation is applied [20].

\section{TRANSMisSion PERFORMANCE}

In this section, we evaluate the influence of fiber nonlinearities on 40 Gbaud RZ-DQP-ASK/D8PSK signals. Since the system is operated at a high symbol rate, we expect the influence of intrachannel nonlinearities, particularly intrachannel four-wave mixing (IFWM) to be dominant [21], [22]. IFWM originates from a nonlinear interaction induced by the overlapping pulses that are dispersively broadened during the 
a)

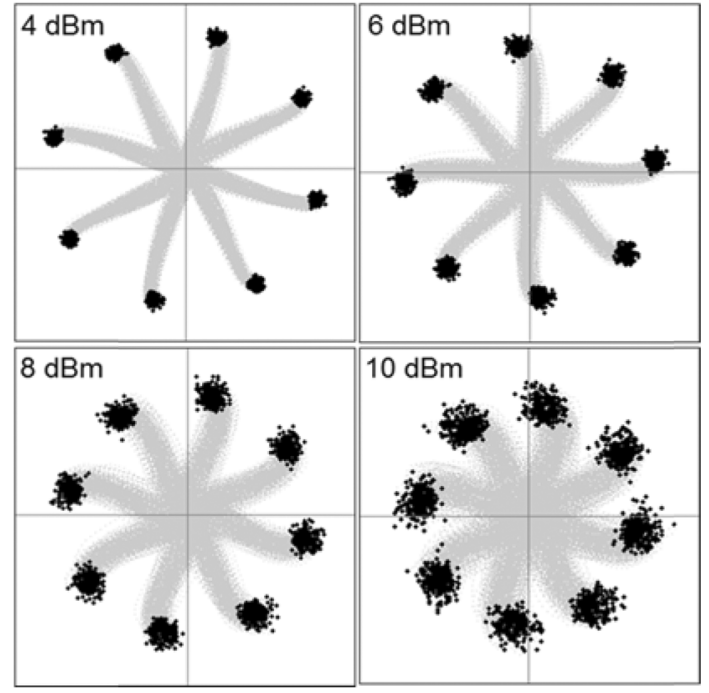

b)

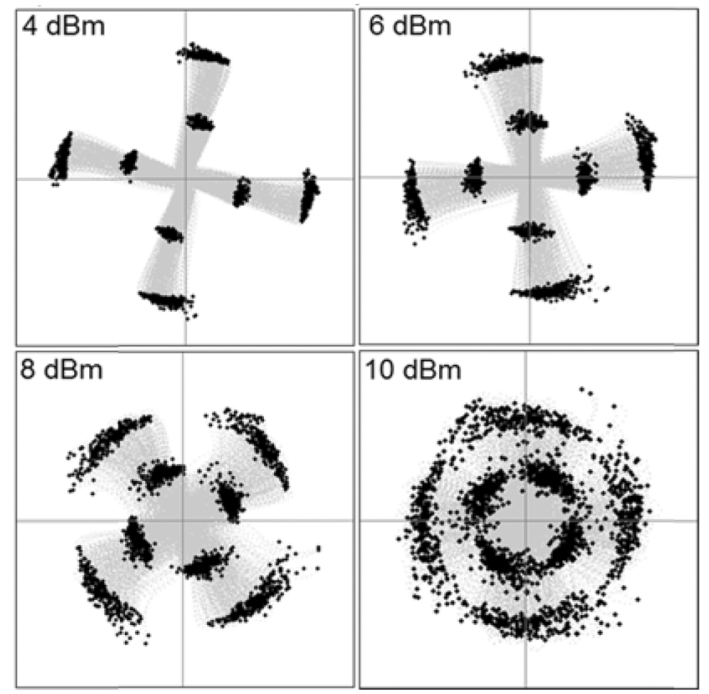

Fig. 9. Simulated noise-free constellations of (a) 40 Gbaud RZ-D8PSK and (b) RZ-DQP-ASK $(\eta=6.88 \mathrm{~dB})$ over a $320 \mathrm{~km}$ link. The launch power was adjusted between $4 \mathrm{dBm} 10 \mathrm{dBm}$.

propagation. Such an interaction give rises to nonlinear transfer of energy, of which the amplitude and phase are determined by those of the overlapping pulses. This transferred energy then interferes coherently with the existing pulses causing fluctuations in both phase and amplitude [23]. Here, we use the signal constellation and the BER to observe and quantify such degradation.

\section{A. Signal Evolution in Nonlinear Transmission}

To understand the significance of fiber nonlinearities on D8PSK and DQP-ASK signals, one should begin by observing the nonlinear evolution of the lower-order formats. Fig. 8 presents the evolution of the simulated noise-free 40 Gbaud RZ-DBPSK and RZ-DQPSK constellations in the transmission limited by fiber nonlinear effects. In this plot, the signals are transmitted over $320 \mathrm{~km}$ of the periodically compensated link as described in Section III with the launch power of $2 \mathrm{dBm}$ and a)
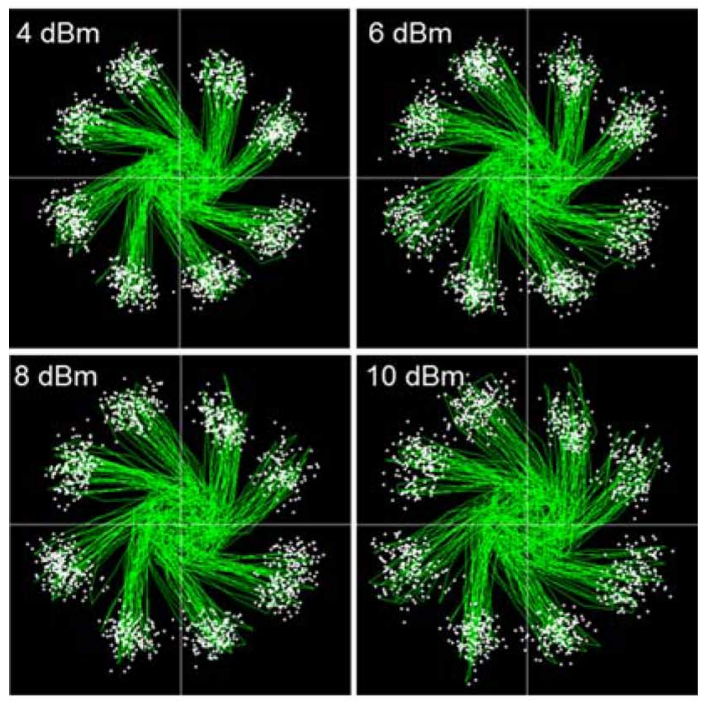

b)
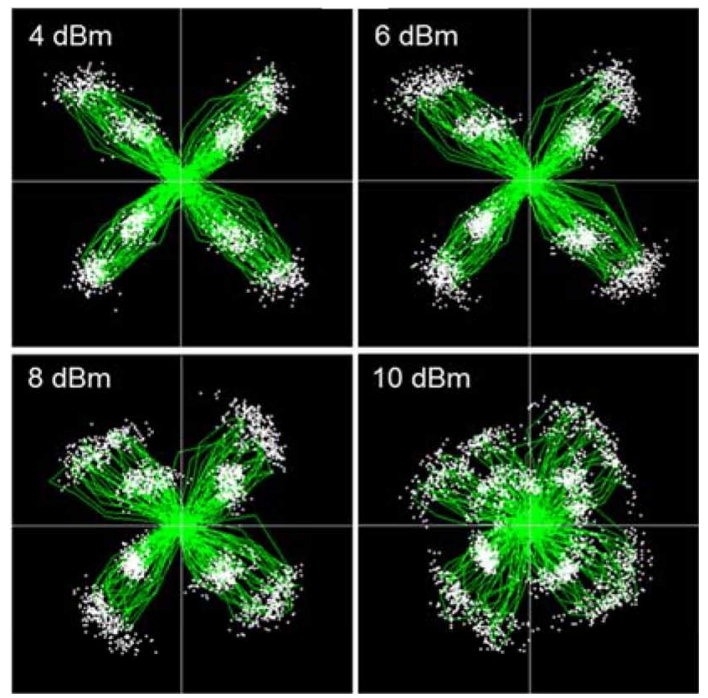

Fig. 10. Measured constellations of 40 Gbaud (a) RZ-D8PSK and (b) RZ-DQP-ASK $(\eta=4.7 \mathrm{~dB})$ over a $320 \mathrm{~km}$ link. The launch power was adjusted between $4 \mathrm{dBm}$ with the corresponding OSNRs ranging from $27.4 \mathrm{~dB}$ to $33.1 \mathrm{~dB}$.

$8 \mathrm{dBm}$. The plots illustrate the nonlinear distortion in both amplitude and phase directions due to intrachannel nonlinearities, which alter the symbol points of DBPSK into a "T" shape while spreading those of DQPSK more uniformly. The " $\mathrm{T}$ " shape in the case of DBPSK has previously been reported and discussed in [24]. The circular shape formed in the case of DQPSK is due to its additional phase states (compared with DBPSK), which in turn provide more degrees-of-freedom of the resultant phase and amplitude of the IFWM.

The uniform spreading of symbol points is also seen in the simulated noise-free 40 Gbaud RZ-D8PSK constellations plotted in Fig. 9(a). In this case, the spreading is rounder since the signal exploits twice the phase states compared to DQPSK.

A different spreading is however observed in Fig. 9(b), which depicts the simulated noise-free 40 Gbaud RZ-DQP-ASK constellations over $320 \mathrm{~km}$ with four different launch powers. In 

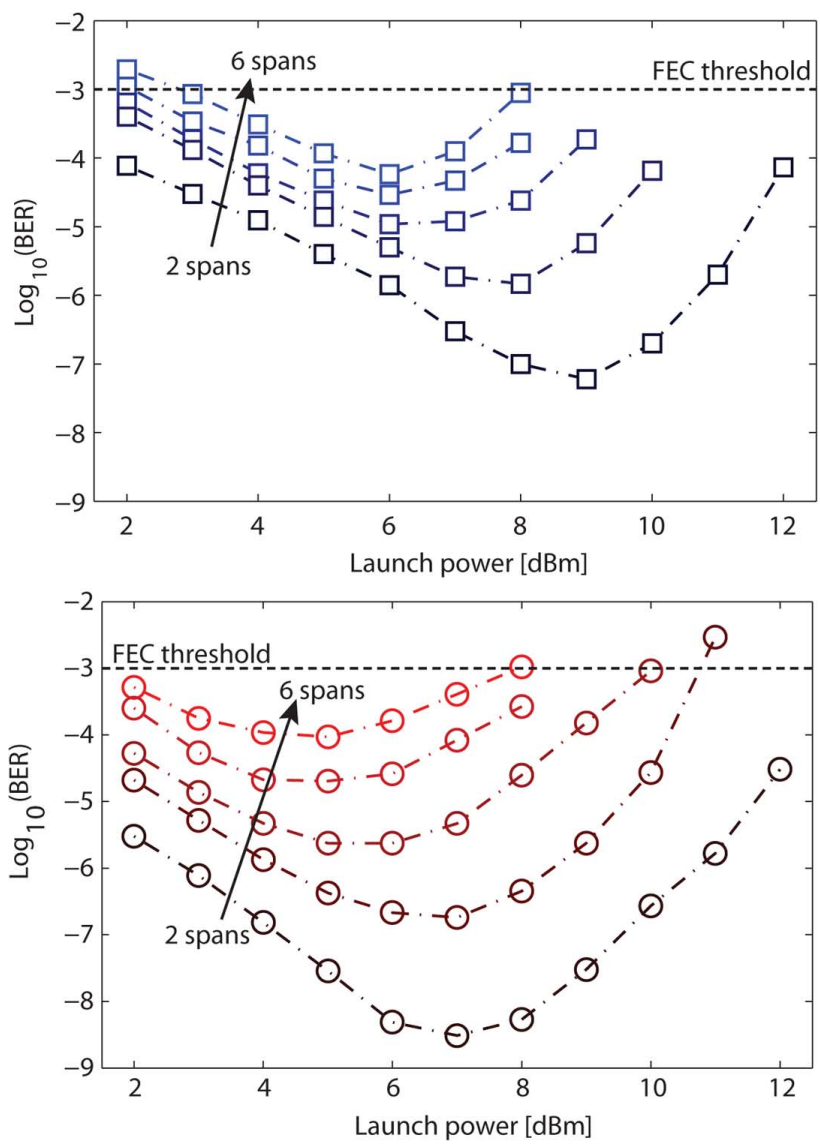

Fig. 11. Experimental BER as a function of the launch power of $40 \mathrm{Gbaud}$ (top) RZ-D8PSK and (bottom) RZ-DQP-ASK over (up to) 6 spans.

these plots, the signal degradation caused by IFWM as well as significant phase distortion (compared to DQPSK) is clearly seen. We attribute such distortion to the additional amplitude modulation, that is, the symbols surrounded by many low-intensity neighbors will experience small spreading due to low nonlinear transfer of energy while those with many of high-intensity neighboring symbols will be severely distorted due to high nonlinear energy transfer.

The numerical observations are well supported by the measured 40 Gbaud RZ-D8PSK and RZ-DQP-ASK constellations over the same distance $(320 \mathrm{~km})$ from the actual system in Fig. 10. The results show high consistency to those obtained from the simulation, validating the accuracy of the simulated system. Compared with those from the simulation, the measured constellations of the two formats however exhibit larger phase and amplitude spreading, which is due to the accumulated ASE noise generated from the in-line EDFAs. It should be noted that, similarly to those in Fig. 2, the plots in Fig. 10 were coherently sampled constellations without applying equalization.

\section{B. Experimental BER Results}

The signal degradation due to fiber nonlinearities can also be observed from the BER. In Fig. 11, we plot the experimental BER of the 40 Gbaud RZ-D8PSK and RZ-DQP-ASK over (up to) 6 spans as a function of the launch power. Note that for the DQP-ASK transmission, the intensity ratio was kept equal to

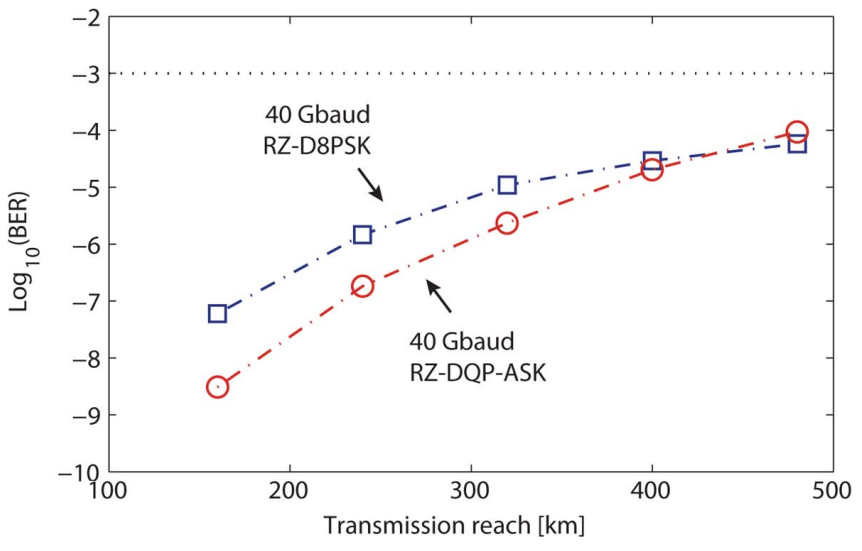

Fig. 12. Experimental BER performance (at optimal launch power) as a function of the transmission distance.

the optimized values obtained in the back-to-back configuration ( $6.8 \mathrm{~dB}$ numerically and $4.7 \mathrm{~dB}$ experimentally).

As expected, each curve identifies an optimal launch power, which is a trade-off between noise and fiber nonlinearities. At low BER $(<10-5)$, a strong dependency of the optimal launch power on the number of spans is clearly seen. However, such a dependency is obscured at high BER by other sources of error such as noisy driving signals and receiver imperfections. In the case of D8PSK, we notice an approximate $2 \mathrm{~dB}$ higher optimal launch power than that of DQP-ASK. We also observe that the BER performance decreases more rapidly with the number of spans in the case of DQP-ASK. These findings indicate that, similarly to the constellation observation, D8PSK is more resilient against fiber nonlinearities.

To summarize the results in Fig. 11, we plot the minimum BER (at the optimal launch power) as a function of the transmission distance in Fig. 12. The plot shows that DQP-ASK outperforms D8PSK at short distances due to its superior back-to-back sensitivity. However, as the reach increases, its performance gradually approaches that of D8PSK due to nonlinear effects and eventually becomes worse at $480 \mathrm{~km}$.

\section{Numerical BER Results}

Fig. 13 presents the numerical BER performance of the two signals as a function of the launch power over (up to) 20 spans. Compared to the experiment, however, the signals were evaluated over longer distances. This is because, as described in Section IV, the noise and data jitter originating from driving signal generation are not included in the simulated system. The curves, thus, illustrate a fundamental limit of the two signals in nonlinear transmission.

Nevertheless, regardless the difference in the distance, we observe similarities between the numerical curves and those (at high BER) obtained experimentally. Firstly, the optimal launch power in the case of DQP-ASK is approximately $2 \mathrm{~dB}$ lower than that of D8PSK. Secondly, the performance at the optimal launch decreases more rapidly with the number of span in the case of DQP-ASK.

Finally, a similar conclusion as from the experiment can be drawn from Fig. 14, that is, DQP-ASK gives a superior performance at shorter distances but becomes worse after a cer- 

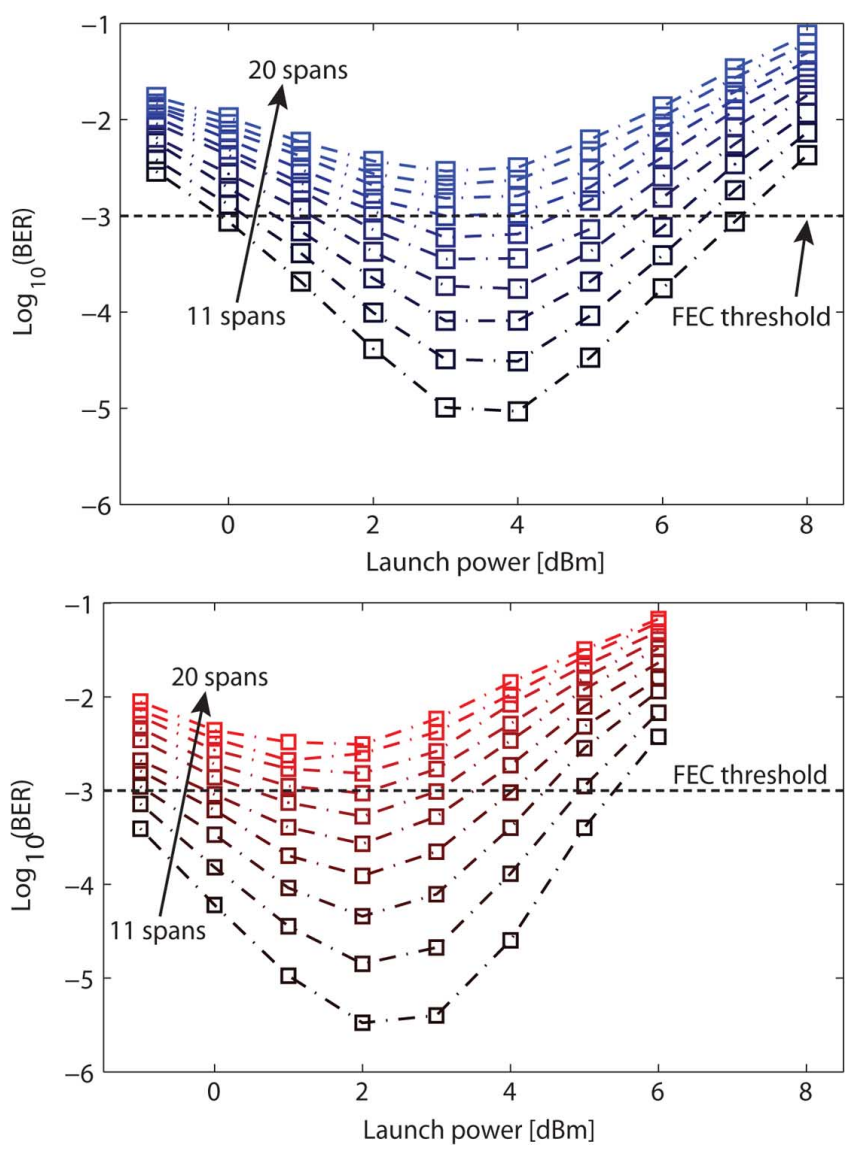

Fig. 13. Numerical BER as a function of the launch power of 40 Gbaud (top) RZ-D8PSK and (bottom) RZ-DQP-ASK over (up to) 20 spans.

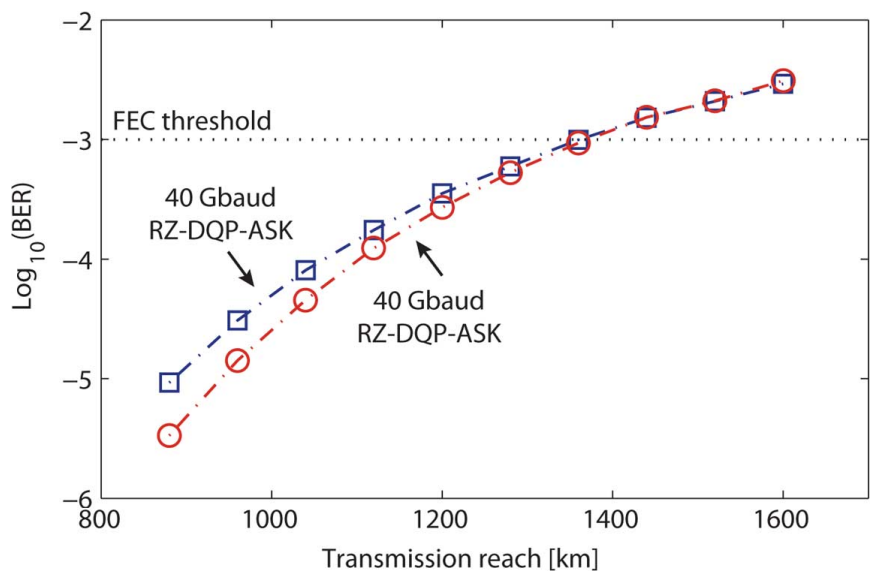

Fig. 14. Numerical BER performance (at optimal launch power) as a function of the transmission distance.

tain distance (in this case, $1500 \mathrm{~km}$ ). It should be noted that although the numerical distance at which D8PSK outperforms DQP-ASK differs from that obtained experimentally by factor of 3.8, it is, indeed, consistent with the difference between the numerical and the experimental back-to-back OSNR requirements. This implies that if all imperfections of the driving signal are included in the numerical model, the same curves should be obtained from the simulation and the experiment.

\section{CONCLUSION}

In this work, the first comprehensive performance comparison between 40 Gbaud RZ-D8PSK and RZ-DQP-ASK in back-to-back and nonlinear transmission is presented. We find that, in the experimental back-to-back transmission, DQP-ASK required approximately $2 \mathrm{~dB}$ lower OSNR than D8PSK for $\mathrm{BER}=10^{-3}$, which leads to superior performance over short transmission distances (up to $400 \mathrm{~km}$ experimentally). However, since D8PSK is more resilient to fiber nonlinearities, its BER performance eventually exceeds that of DQP-ASK over long distances (more than $400 \mathrm{~km}$ experimentally). Lastly, our experimental findings are consistent with the analytical and numerical solutions.

\section{ACKNOWLEDGMENT}

The authors would like to thank EXFO Sweden AB for the loan of an optical modulation analyzer.

\section{REFERENCES}

[1] A. H. Gnauck, S. Chandrasekhar, J. Leuthold, and L. Stulz, "Demonstration of $42.7-\mathrm{Gb} / \mathrm{s}$ DPSK receiver with 45 photons/bit sensitivity," IEEE Photon. Technol. Lett., vol. 15, no. 1, pp. 99-101, Jan. 2003.

[2] B. Koch, R. Noé, D. Sandel, V. Mirvoda, V. Filsinger, and K. Puntsri, "200-Gb/s, 430-km PDM-RZ-DQPSK (4 bit/symbol) transmission with $10 \mathrm{krad} / \mathrm{s}$ endless polarization tracking," in Proc. Opt. Fiber Commun. Conf., San Diego, CA, 2010, Paper OThD4.

[3] B. Koch, R. Noe, V. Mirvoda, D. Sandel, V. Filsinger, and K. Puntsri, "40-krad/s polarization tracking in 200-Gb/s PDM-RZ-DQPSK transmission over 430 km," IEEE Photon. Technol. Lett., vol. 22, no. 9, pp. 613-615, May 2010.

[4] T. Tokle, M. Serbay, Y. Geng, J. B. Jensen, W. Rosenkranz, and P. Jeppesen, "Penalty-free transmission of multilevel $240 \mathrm{Gbit} / \mathrm{s}$ RZ-DQPSK-ASK using only $40 \mathrm{Gbit} / \mathrm{s}$ equipment," in Proc. Eur. Conf. Opt. Commun., Gasglow, U.K., 2005, Paper Th.4.1.6.

[5] T. Tokle, M. Serbay, J. B. Jensen, Y. Geng, W. Rosenkranz, and P. Jeppesen, "Investigation of multilevel phase and amplitude modulation formats in combination with polarization multiplexing up to 240 Gbit/s," IEEE Photon. Technol. Lett., vol. 18, no. 20, pp. 2090-2092, Oct. 2006.

[6] E. Tipsuwannakul, M. Sköld, M. Karlsson, and P. A. Andrekson, "Transmission of $240 \mathrm{~Gb} / \mathrm{s}$ PM-RZ-D8PSK over $320 \mathrm{~km}$ in $10 \mathrm{~Gb} / \mathrm{s}$ NRZ-OOK WDM system," in Proc. Opt. Fiber Commun. Conf., San Diego, CA, 2010, Paper OMJ2.

[7] E. Tipsuwannakul, M. N. Chughtai, M. Forzati, J. Mårtensson, P. A. Andrekson, and M. Karlsson, "Influence of self- and cross-phase modulation on 40 Gbaud dual polarization DQPSK/D8PSK signals in $10 \mathrm{Gbit} / \mathrm{s}$ OOK WDM systems," Opt. Exp., vol. 18, no. 23, pp. 24178-24188, 2010.

[8] K. Sekine, N. Kikuchi, S. Sasaki, S. Hayase, C. Hasegawa, and T. Sugawara, "40 Gbit/s, 16-ary (4 bit/symbol) optical modulation demodulation scheme," Electron. Lett., vol. 41, no. 7, 2005.

[9] M. Ohm and J. Speidel, "Receiver sensitivity, chromatic dispersion tolerance and optimal receiver bandwidths for $40 \mathrm{~Gb} / \mathrm{s} 8$-level optical ASK-DQPSK and optical 8-DPSK," in Proc. 6th Conf. Photon. Netw., Leipzig, Germany, May 2005, pp. 211-217.

[10] E. Tipsuwannakul, M. Karlsson, E. Agrell, and P. Andrekson, "Performance comparison between $120 \mathrm{Gbit} / \mathrm{s}$ RZ-DQP-ASK and RZ-D8PSK over a 480 km link," in Proc. Opt. Fiber Commun. Conf., Los Angeles, CA, 2011, Paper OMI5.

[11] M. S. Alfiad, D. van den Borne, T. Wuth, M. Kuschnerov, B. Lank1, C. Weiske, E. de Man, A. Napoli, and H. de Waardt, "111-Gb/s POLMUX-RZ-DQPSK transmission over $1140 \mathrm{~km}$ of SSMF with 10.7-Gb/s NRZ-OOK neighbours," in Proc. Eur. Conf. Opt. Commun., Brussels, Belgium, 2008, Paper Mo.4.E.2.

[12] Y. K. Lizé1, L. Christen, X. Wu, J.-Y. Yang, S. Nuccio, T. Wu, A. E. Willner, and R. Kashyap, "Free spectral range optimization of return-to-zero differential phase shift keyed demodulation in the presence of chromatic dispersion," Opt. Exp., vol. 15, no. 11, pp. 6817-6822, 2007.

[13] G. P. Agrawal, Nonlinear Fiber Optics, 4th ed. New York: Academic, 2007, ch. 4. 
[14] C. Behrens, R. I. Killey, S. J. Savory, M. Chen, and P. Bayvel, "Nonlinear distortion in transmission of higher order modulation formats," IEEE Photon. Technol. Lett., vol. 22, no. 15, pp. 1111-1113, 2010

[15] R. F. Pawula, S. Rice, and J. H. Roberts, "Distribution of the phase angle between two vectors perturbed by Gaussian noise," IEEE Trans. Commun., vol. COM-30, no. 8, pp. 1828-1841, Aug. 1982.

[16] J. Proakis, Digital Communications, 4th ed. : McGraw-Hill, 2000

[17] C. Kim and G. Li, "Direct-detection optical differential 8-level phase-shift keying (OD8PSK) for spectrally efficient transmission," Opt. Exp., vol. 12, no. 15, pp. 3415-3421, 2004.

[18] C. Kim, G. Goldfarb, Y. Han, Z. Li, X. Li, and G. Li, "WDM transmission over $320 \mathrm{~km}$ EDFA-amplified SSMF using $30 \mathrm{~Gb} / \mathrm{s}$ return-to-zero optical differential 8-level phase-shift-keying (OD8PSK)," Opt. Exp., vol. 13, no. 11, pp. 4044-4049, 2004.

[19] H. Yoon, D. Lee, and N. Park, "Performance comparison of optical 8-ary differential phase-shift keying systems with different electrical decision schemes," Opt. Exp., vol. 13, no. 2, pp. 371-376, 2005.

[20] X. Liu, S. Chandrasekhar, A. Gnauck, and X. Wei, "OSNR margin assessment for optical transmissions in the nonlinear Regime with forward error correction," in Proc. Opt. Fiber Commun. Conf., Atlanta, GA, 2003, Paper ThN2.

[21] R.-J. Essiambre, G. Raybon, and B. Mikkelsen, , I. Kaminow and T. Li, Eds., Pseudo-Linear Transmission of High-Speed TDM Signals: 40 and $160 \mathrm{~Gb} / \mathrm{s}$. New York: Academic, 2002, pp. 232-304.

[22] R.-J. Essiambre, B. Mikkelsen, and G. Raybon, "Intra-channel crossphase modulation and four-wave mixing in high-speed TDM systems," Electron. Lett., vol. 35, pp. 1576-1578, 1999.

[23] A. Mecozzi, M. Tabacchiera, F. Matera, and M. Settembre, "Dispersion management in phase modulated optical transmission systems," in Proc. Eur. Conf. Opt. Commun., Torino, Italy, 2010, Paper Mo.2.C.2.

[24] K.-P. Ho, Phase-Modulated Optical Communication Systems. Berlin, Germany: Springer, 2005, ch. 7.

Ekawit Tipsuwannakul was born in Bangkok, Thailand, in 1982. He received the B.Sc. degree in electrical engineering from Chulalongkorn University, Bangkok, Thailand, in 2004, and the M.Sc. degree in photonics from the Royal Institute of Technology (KTH), Stockholm, Sweden, in 2008. He is currently working toward the Ph.D. degree at Chalmers University of Technology, Göteborg, Sweden.

During 2004-2006, he served as a Test Engineer for the Optical Transmitter/Receiver Manufacturing Group, Fabrinet Co. Ltd, Bangkok, Thailand He later joined Svedice/Northlight AB, Stockholm, Sweden, in 2006, where he spent two years involving in the system designs for the high-speed optical transmitter/receiver characterizations. His research is devoted to high-speed optical transmission systems using multilevel modulation formats and all-optical signal processing.

Pontus Johannisson received the Ph.D. degree from Chalmers University of Technology, Göteborg, Sweden, in 2006. His thesis was focused on nonlinear intrachannel signal impairments in optical fiber communications systems.

In 2006, he joined the research institute IMEGO, Göteborg, Sweden, where he worked with digital signal processing for inertial navigation with MEMS-based accelerometers and gyroscopes. In 2009, he joined the Photonics Laboratory, Chalmers University of Technology, where he is currently an Assistant Professor. His research interests include nonlinear effects in optical fibers and digital signal processing in coherent optical receivers.

Mats Sköld received the M.Sc. and Ph.D. degrees in electrical engineering from Chalmers University of Technology, Göteborg, Sweden, in 2001 and 2008, respectively. The title of his Ph.D. thesis was "Signal Characterization in Optical Networks" and was focusing on methods of analyzing optical signal properties in intensity, phase, and polarization.

After receiving his Ph.D. degree, he continued his research activities on modulation formats, optical coherent systems, characterization of optical signals, and optical network monitoring at the Photonics Laboratory at Chalmers University of Technology. He is the author and coauthor of more than 30 journal articles and conference contributions. In 2008, he joined the Chalmers spin-off company PicoSolve, developing optical sampling systems. Since 2009, he has been a Research Scientist with EXFO Sweden, Göteborg, working with development of test and measurement equipment for optical communication systems.
Erik Agrell received the M.S. degree in electrical engineering and Ph.D. degree in information theory from Chalmers University of Technology, Sweden, in 1989 and 1997, respectively.

From 1988 to 1990, he was with Volvo Technical Development as a Systems Analyst and, from 1990 to 1997, with the Department of Information Theory, Chalmers University of Technology, as a Research Assistant. During 1997-1999, he was a Postdoctoral Researcher with the University of Illinois at Urbana-Champaign and the University of California, San Diego. In 1999, he joined the faculty of Chalmers University of Technology, first as an Associate Professor and, since 2009, as a Professor in Communication Systems. His research interests belong to the fields of information theory, coding theory, and digital communications, and his favorite applications are found in optical communications. More specifically, his current research includes bit-interleaved coded modulation and multilevel coding, polarization-multiplexed coding and modulation, transmission over the optical intensity channel, phase noise mitigation, and bit-to-symbol mappings in coded and uncoded systems.

Prof. Agrell served as Publications Editor for the IEEE TRANSACTIONS ON INFORMATION THEORY from 1999 to 2002.

Magnus Karlsson received the Ph.D. degree in electromagnetic field theory from Chalmers University of Technology, Göteborg, Sweden, in 1994. The title of his Ph.D. thesis was "Nonlinear propagation of optical pulses and beams."

Since 1995, he has been with the Photonics Laboratory at Chalmers University of Technology, first as an Assistant Professor and, since 2003, as a Professor of photonics. He has authored or coauthored over 190 scientific journal and conference contributions, served as guest editor for the Journal of Lightwave Technology, and is currently associate editor of Optics Express. His research has been devoted to a variety of aspects of fiber optic communication systems, in particular transmission effects such as fiber nonlinearities and polarization effects, but also applied issues such as high-capacity data transmission and all-optical switching. Currently he is devoted to parametric amplification, multilevel modulation formats, and coherent transmission in optical fibers.

Dr. Karlsson has served in the technical committees for the Optical Fiber Communication Conference (OFC) (2009 as subcommittee chair), and the Asia Communications and Photonics Conference (ACP, formerly APOC).

Peter A. Andrekson (F'06) received the Ph.D. degree from Chalmers University of Technology, Göteborg, Sweden, in 1988.

After approximately three years with AT\&T Bell Laboratories, Murray Hill, NJ, during 1989-1992, he returned to Chalmers University of Technology, where he is now a full Professor with the Department of Microtechnology and Nanoscience. He was Director of Research at Cenix Inc., Allentown, PA, during 2000-2003 and with the newly established Center for Optical Technologies, Lehigh University, Bethlehem, PA, during 2003-2004. His research interests include nearly all aspects of high-speed and high-capacity fiber communications such as optical amplifiers, nonlinear pulse propagation, all-optical functionalities, and very high speed transmission. He is Cofounder of the optical test and measurement company Picosolve Inc., now part of EXFO, Göteborg, and where he is a Director of EXFO Sweden AB. He is the author and coauthor of over 300 scientific publications and conference papers in the area of optical communications, more than sixty of which were invited papers at leading international conferences and journals. He also holds several patents.

Dr. Andrekson is a Fellow of the Optical Society of America. He is or has served on several technical program committees, including OFC and ECOC, and as international project and candidate evaluator, and has also served twice as an expert for the evaluation of the Nobel Prize in Physics. He was an associate editor for the IEEE PHOTONICS TECHNOLOGY LETTERS during 2003-2007. In 1993, he was awarded a price from the Swedish government research committee for outstanding work performed by young scientists, and in 2000 he was awarded the Telenor Nordic research award for his contribution to optical technologies. 